\title{
BatNet: A 6LoWPAN-Based Sensors and Actuators Network
}

\author{
Guillermo del Campo, Eduardo Montoya, Jorge Martín, Igor Gómez, \\ and Asunción Santamaría
}

\begin{abstract}
Improving energy efficiency in buildings is one of the goals of the Smart City initiatives and a challenge for the European Union. This paper presents a 6LoWPAN wireless transducer network (BatNet) as part of an open energy management system. This network has been designed to operate in buildings, to collect environmental information (temperature, humidity, illumination and presence) and electrical consumption in real time (voltage, current and power factor). The system has been implemented and tested in the Energy Efficiency Research Facility at CeDInt-UPM.
\end{abstract}

Keywords: Home Automation, Wireless Transducer Network, 6LoWPAN, Smart Meter, Energy Efficiency in Buildings.

\section{Introduction}

Smart Cities aim to improve citizens' life by means of the integration of new architectonic elements, technological innovations and Information and Communication Technology (ICT) infrastructures.

Energy consumption in buildings, which is one of the cornerstones of the Smart Cities, accounts for between $20 \%$ and $40 \%$ of the total energy used in developed countries [1]. Gathering information related to the use of energy made by devices, users and systems within a building is useful to improve the efficiency of electricity distribution systems, since it provides accurate information to avoid peaks and dips in energy demand.

Real time metering systems of electric line parameters (such as current, voltage or real power) provide accurate information related to the building electrical consumption. This information is essential to improve electricity generation and distribution in a smart grid. Most of the distribution board metering devices proposed so far only measure electric current and consequently the power consumption is calculated assuming a constant voltage from the electric line [2]. Other devices measure the line voltage as well but just on one electric line.

In order to solve these problems and serve as a basis for smart cities we propose an energy management system for buildings. The system comprises a management 
platform and wireless transducer network (WTN). The system description is presented in Section 2 while the WTN architecture, elements and implementation are described in Section 3.

\section{System Description}

The building energy management system proposed consists of a management platform (Bat-MP) and a wireless transducer network (BatNet) which allows real time metering of electric line, ambient parameters and electrical loads and control of devices.

The BatNet is connected to the Bat-MP through one of its nodes which acts as coordinator. The BatNet sends data from sensors (temperature, illumination, humidity or presence) to the Bat-MP, which in return sends control orders to the actuators (HVAC -heating, ventilation, and air conditioning-, lamps or blinds). The Bat-MP provides locally a set of services (monitoring, scheduling...) while its connection to Internet enhances the number and power of services (remote server). Besides, it enables the compilation of useful external information as weather prediction or user location and the communication with the utility to provide metering information or to allow the implementation of demand response services (Fig. 1).

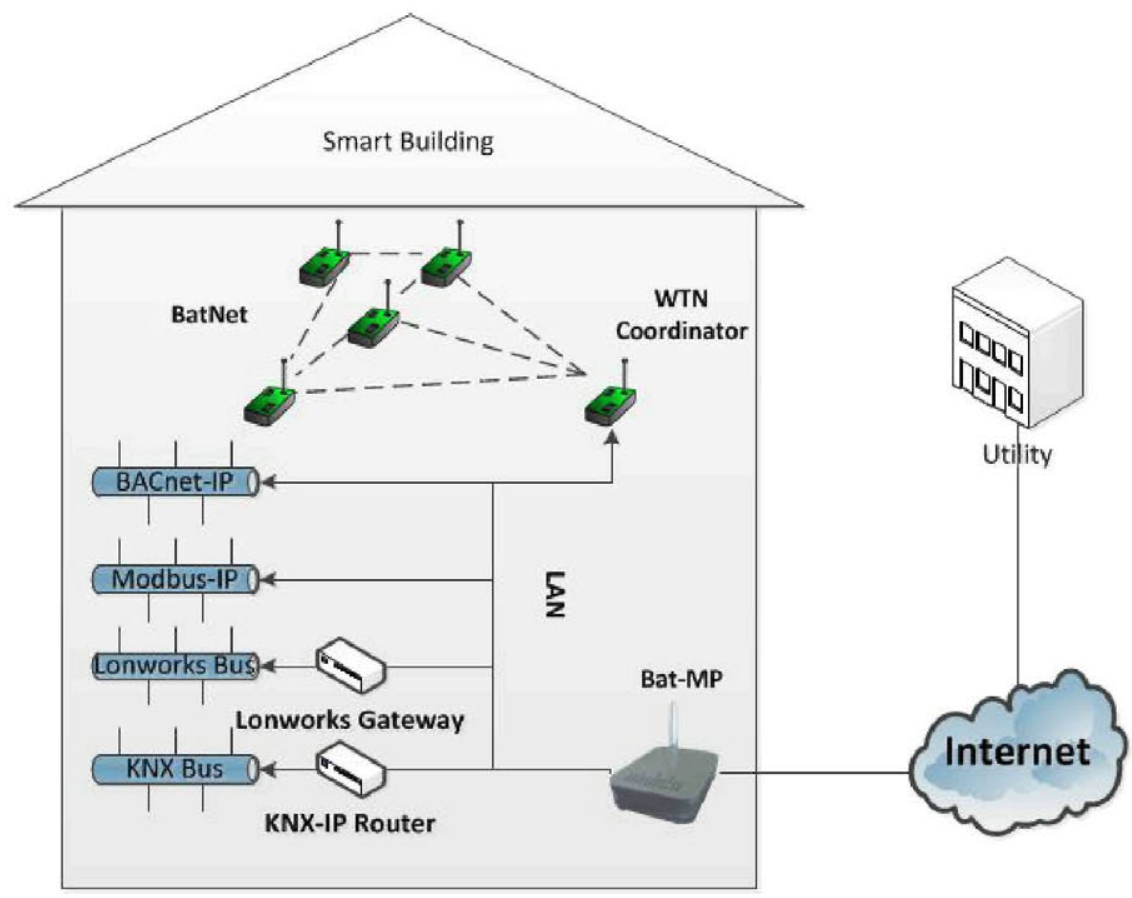

Fig. 1. Structure of the Wireless System for Energy Efficiency in Buildings 


\section{$3 \quad$ BatNet (Wireless Transducer Network)}

In order to provide the building management platform with relevant data (such as power measurement) and to enable different devices control, a wireless transducer network (BatNet) has been designed and implemented. The main characteristics of BatNet, focused on avoiding the traditional systems limitations in terms of cost, interoperability, power consumption and collaboration, are:

- 6LoWPAN-based network: 6LoWPAN (IPv6 over Low power Wireless Personal Area Networks) working group from the IETF describes the adaptation of IPv6 packets to IEEE 802.15.4 based networks. 6LoWPAN uses physical and data link layers based on IEEE 802.15.4 protocol (like other low power wireless communications as ZigBee or MiWi) and uses IPv6 (where a single IP address is assigned to each BatNet device) for the network layer [3].

- CoAP communication interfaces: CoAP (Constrained Application Protocol) (currently an IETF draft) is a specific web transfer protocol designed to be used with constrained nodes and networks. This protocol is simple and easy to implement [4].

- Contiki Operating System: the open source operating system from SICS enables multitasking and implements both IPv6 and IEEE 802.15.4 standards under low-capacitance hardware requirements [5].

BatNet is based on a modular functional architecture, which eases the development of the different transducers and the integration of external devices. Each node of the BatNet comprises a processing and communications module (BatMote) plus a transducer module. Transducer modules implemented are a power meter (BatMeter), ambient sensors (BatSense), power switch (BatPlug/BatSwitch), light dimmer (BatDimm) and blinds control (BatBlind).

\subsection{BatMote: Processing and Communications Module}

There are several processing and communication tasks common to every BatNet device, with independency of the node type (transducer, coordinator or repeater). Gathering these functions in a single device simplifies modules programming and enables and makes it easier and more efficient their implementation, due to the modular architecture of devices. First, the use of available commercial boards such as Zigduino [6] or Zolertia Z1 [7] was considered, but BatNet devices have two requirements that are a must: reduced size and the possibility of integrating a wide variety of sensors. To fulfill these requirements a low-cost and low-power processing and communication module has been designed and implemented: the BatMote (see Fig. 2).

The core of the BatMote is the ATmega 128RFA1 from Atmel Corporation. The ATmega 128RFA1 is a single chip microprocessor integrating an IEEE 802.15.4 transceiver, which allows a noticeable reduction of the hardware size. Besides, the ATmega 128RFAl provides 8 ADC channels (10 bits resolution), allowing data collection from different sources simultaneously. 


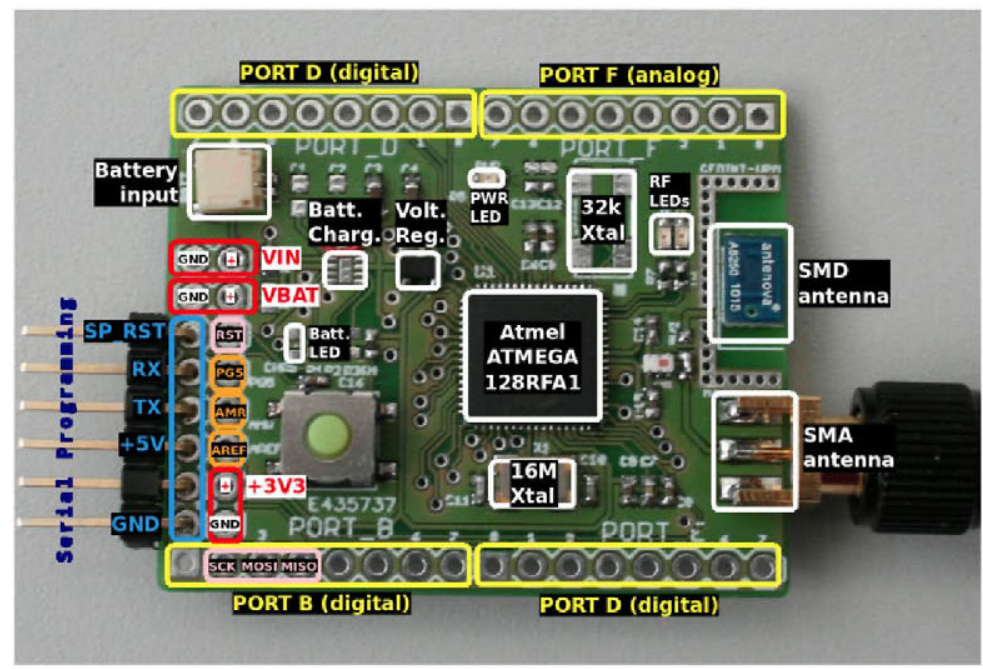

Fig. 2. BatMote

BatMote has been designed to allow easy connection of transducer modules, enable different installation locations and provide multiple data transmission intervals and sleep mode functions.

BatMote can be powered by batteries or other external power sources (3.3-12 V). It also includes a battery charger allowing the batteries to recharge through a USB port.

BatNet devices will be located at electrical distribution boards, room ceilings and walls, where there could be a lack of space or where small and discreet devices would be preferable; hence size and shape of the antenna could be critic. BatMote is designed to be used with both SMD (Surface-mount technology) and SMA (SubMiniature version A) antennas.

The communication range of the BatMote has been measured within CeDInt-UPM building. In a single floor, the coverage range is about 30 meters. In upper and lower floors, the coverage is nearly 20 meters. Therefore, communication range is not a problem since BatNet devices can act as repeaters.

\subsection{Transducer Modules}

According to the functionality desired and the signal type (light, temperature, humidity or voltage) different sensor and actuator modules have been designed:

\section{BatSense}

It is an ambient Multi-Sensor module which includes temperature, humidity, illumination and presence sensors together in a single device. Other ambient parameters (such as $\mathrm{CO} 2$ or noise) sensors can be added since extra pads and pins are still available. 


\section{BatPlug and BatSwitch}

BatPlug controls the ON/OFF status of appliances or devices connected to a socket while measuring the power consumption. A TRIAC (Triode for Alternating Current) deals with the $\mathrm{ON} / \mathrm{OFF}$ switching. Power measurement is realized by monitoring both current (through a Hall Effect sensor) and voltage. BatSwitch can measure and control up to five appliances simultaneously.

\section{BatMeter: Power Meter Module}

It is a power meter sensor designed to be installed in the distribution board as a rail DIN (Deutsches Institut für Normung) device. It calculates simultaneously the electrical power and energy consumption of a maximum of seven electric lines (within the same phase) from real time voltage and current measurements (see Fig. 3).

The electric line voltage passes through a voltage transformer with two outputs. One output is used to power the BatMote passing through a voltage divider. The second output is used to measure the voltage signal. Using split core current transformers (SCT), up to seven different lines can be monitored. The measured analog signals enter the processor through the ADCs.

The BatMote processor has been programmed to calculate different electric power parameters. The libraries from the Open Energy Monitor project ${ }^{1}$ have been used and modified to run under the Contiki OS, in such a way that the device is able to measure more than one line at once.

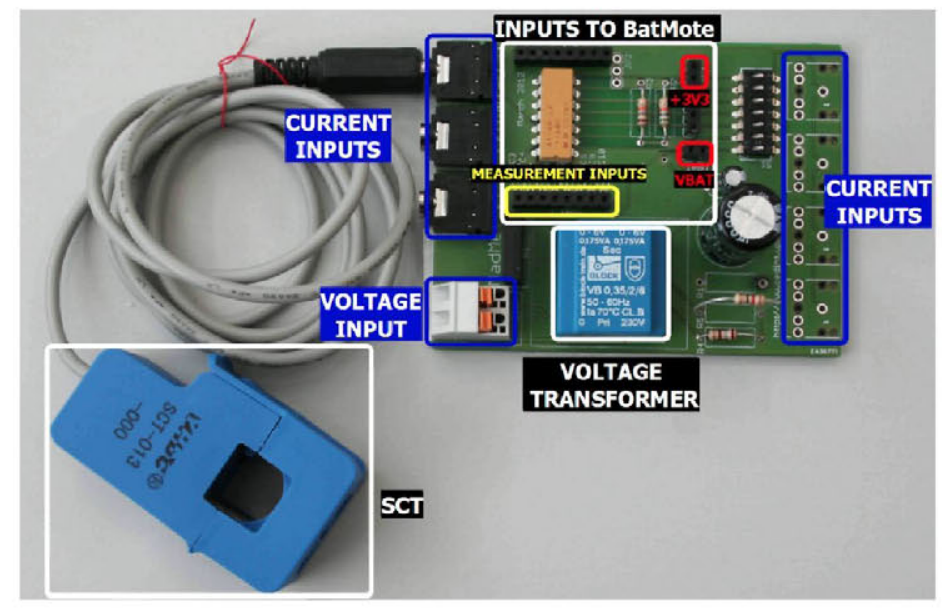

Fig. 3. BatMeter and SCT

The power and related parameters calculation works as follows:

1. The BatMeter measures continuously instantaneous voltage and current, and the BatMote calculates instantaneous power.

\footnotetext{
${ }^{1}$ Open Energy Monitor project: www . openenergymonitor .org
} 
2. Every 20 electric signal periods (400ms) average voltage, current, active power, apparent power and power factor are calculated.

3. Average values are sent every 15 seconds to a BatMote connected to a PC.

The whole smart meter consists of a BatMote and a BatMeter and has been installed in the distribution board at CeDInt-UPM Energy Efficiency Research Facility (EERF) (see Fig. 4). This distribution board has separated lines to independently measure electrical consumption in three illumination groups, two HVAC systems and six appliance lines.

Different lines (Illumination, HVAC and Electric Water Heater) and appliances (Electric Heater and Computer) have been monitored.

To validate the system, energy consumption measures have been compared to those obtained from a commercial static meter installed at the distribution board (Model: Orbis Contax 2511SO). Table 1 shows the energy consumption measurements from different appliances during one hour working time:

Table 1. Energy measurements results

\begin{tabular}{|c|c|c|c|}
\hline \multirow{2}{*}{ Appliance } & \multicolumn{2}{|c|}{ Meter } & \multirow{2}{*}{ Error (\%) } \\
\cline { 2 - 3 } & Orbis Contax 2511SO (Wh) & BatMeter (Wh) & \\
\hline Lights & 310 & 314.6 & 1.48 \\
\hline $\begin{array}{c}\text { Electric Water } \\
\text { Heater }\end{array}$ & 698 & 690.4 & 1.08 \\
\hline Electric Heater & 1810 & 1796.3 & 0.75 \\
\hline Computer & 63 & 60.45 & 4.04 \\
\hline
\end{tabular}

As it can be seen in Table 1, the developed smart meter shows accurate measurements for all the appliances with an error below $5 \%$.

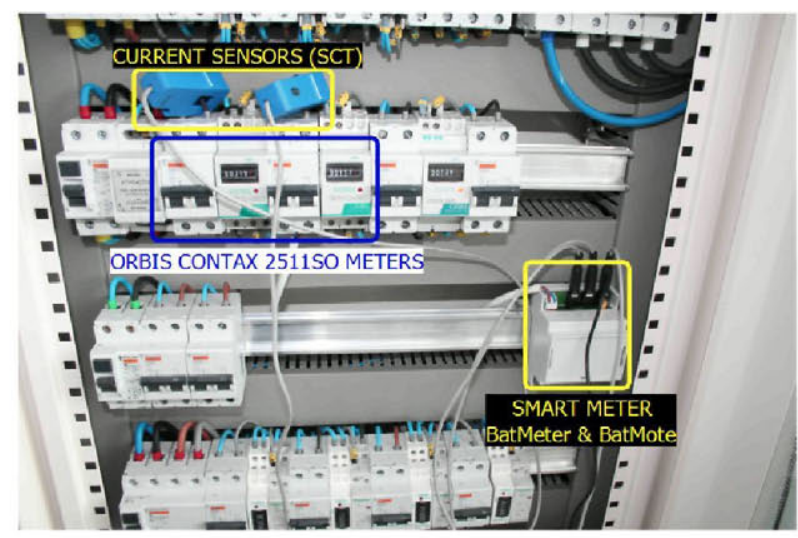

Fig. 4. Smart meter installed at the distribution board 
Figure 5 shows the measured electric parameters (average voltage and average current) for a line where a $\mathrm{PC}$ has been connected. During the time period represented (1 hour), electric voltage varies between $233 \mathrm{~V}$ and $237 \mathrm{~V}$. For long-time monitoring (a 96 hour period) a $228 \mathrm{~V}-243 \mathrm{~V}$ range has been recorded. Measured current is almost constant below $300 \mathrm{~mA}$ for the PC dealing with low load processes during the time period.

Consequently in the current measurements obtained, the active and apparent power do not suffer major oscilations. The power factor oscilates between $90 \%$ and $99 \%$, which matchs the assumed power factor of a PC with an active power factor corrector power supply (95\%). Energy consumption of $60 \mathrm{Wh}$ corresponds to value measures for the expected value for $1.7 \mathrm{GHz}$ Pentium IV computer.
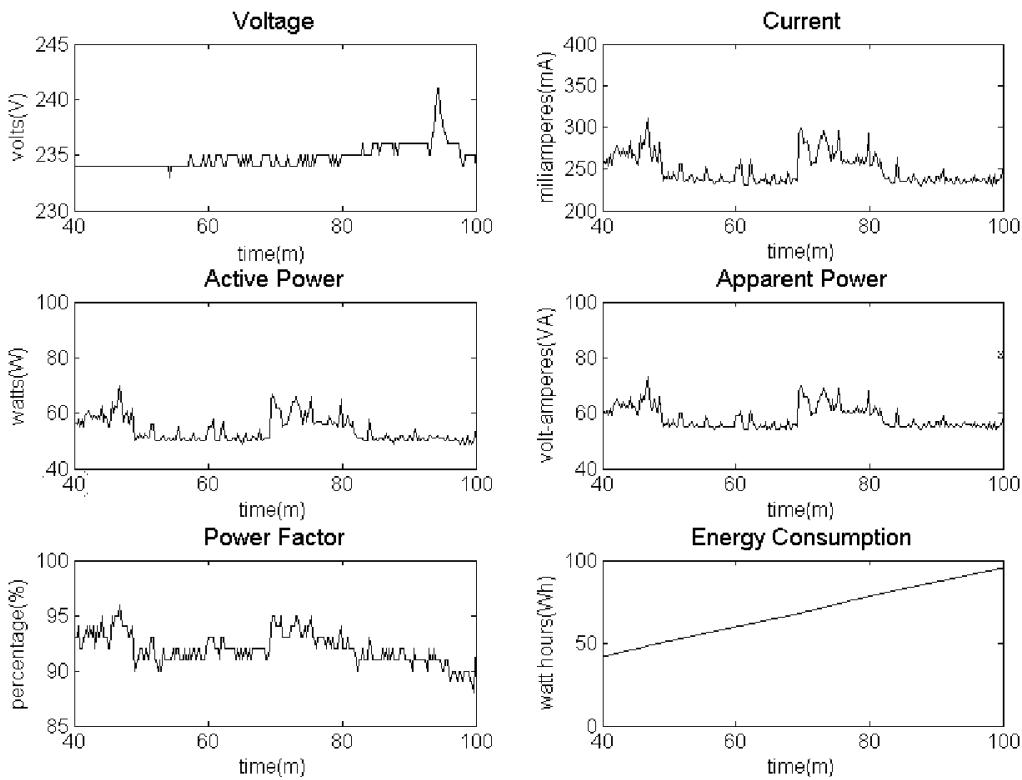

Fig. 5. Voltage and Current measurements and Power (active and apparent), Power Factor and energy calculations for a $\mathrm{PC}$

\section{Conclussions}

This paper presents a wireless transducer network (BatNet) as part of a building energy management system. BatNet design aims to solve current commercial system issues: high cost, not real voltage measurement and limited number of electric lines monitoring.

BatNet comprises ambient sensors, power meters and actuators. The modular architecture design of the network nodes and the use of a common communication and 
processing module (BatMote) allow an easy integration of new sensors and actuators and provide several advantages in terms of design, programming and installation.

Finally, results of real time power consumption measurements realized by the smart meter -composed by the power meter module (BatMeter) and the BatMote- are presented. The smart meter calculates power consumption along with other electric parameters (power factor, active power and apparent power) by real time measuring of both current and voltage.

\section{References}

1. Pérez-Lombard, L., Ortiz, J., Pout, C.: A Review on buildings energy consumption information. In: Energy and Building. Elsevier (March 2007)

2. Ploennigs, J., Ryssel, U., Kabitzsch, K.: Performance Analysis of the EnOcean Wireless Sensor Network Protocol. In: Proc. 20120 IEEE Conference on Emerging Technologies and Factory Automation (ETFA), pp. 1-9 (2010), doi:10.1109/ETFA.2010.5641313,2010

3. Lu, C.-W., Li, S.-C., Wu, Q.: Interconnecting ZigBee and 6LoWPAN wireless sensor networks for smart grid applications. In: Proc. 2011 Fifth International Conference on Sensing Technology (ICST), pp. 267-272 (2011), doi:10.1109/ICSensT.2011.6136979

4. Constrained Application Protocol (CoAP) draft, https://datatracker.ietf.org/doc/draft-ietf-core-coap/

5. Zigduino, http://www. logos-electro.com/zigduino

6. Zolertia Z1, http://wWW. zolertia.com/products/z1

7. Oikonomou, G., Phillips, I.: Experiences from porting the Contiki operating system to a popular hardware platform. In: 2011 International Conference on Distributed Computing in Sensor Systems and Workshops (DCOSS), pp. 1-6 (2011), doi:10.1109/DCOSS. 2011.5982222 